\title{
Combination of Urinary Sodium/Creatinine Ratio and Plasma Brain Natriuretic Peptide Level Predicts Successful Tolvaptan Therapy in Patients With Heart Failure and Volume Overload
}

\author{
Yuichi Sato, ${ }^{1}$ MD, Kaoru DohI, ${ }^{1}$ MD, Kiyotaka Watanabe, ${ }^{1}$ MD, \\ Muneyoshi Tanimura, ${ }^{1}$ MD, Tetsushiro Takeuchi, ${ }^{1}$ MD, Emiyo Sugiura, ${ }^{1}$ MD, \\ Tadafumi Sugimoto, ${ }^{1}$ MD, Naoto Kumagai, ${ }^{1}$ MD, Toru Ogura, ${ }^{2} \mathrm{PhD}$, Shiro NaKamori, ${ }^{1}$ MD, \\ Naoki Fujımoto, ${ }^{3}$ MD, Norikazu Yamada, ${ }^{1} \mathrm{MD}$, and Masaaki ITo, ${ }^{1} \mathrm{MD}$
}

\begin{abstract}
SUMMARY
To evaluate the short-term clinical and hemodynamic effects of tolvaptan therapy and to identify predictors of the therapeutic outcomes, we retrospectively recruited 60 consecutive hospitalized heart failure (HF) patients ( $70 \pm 11$ years) with volume overload. The subjects were divided into two groups on the basis of the changes in HF symptom scores and hemodynamic status assessed by right heart catheterization after tolvaptan therapy (median: 7 days). The majority of patients were successfully treated (group 1). However, $22 \%$ of patients (group 2) were unsuccessfully treated, in whom 1) the HF symptom score worsened or 2) there was a stationary HF symptom score $\geq 6$ points, and mean PCWP $>18$ $\mathrm{mmHg}$ and mean RAP $>10 \mathrm{mmHg}$, after tolvaptan therapy. HF symptom scores, hemodynamic parameters, and plasma brain natriuretic peptide (BNP) level improved in group 1, but all of these parameters remained unchanged in group 2. Lower urine sodium/creatinine ratio $(\mathrm{UNa} / \mathrm{UCr})$ and higher BNP level at baseline were independently associated with unsuccessful tolvaptan therapy, and $\mathrm{UNa} / \mathrm{UCr}$ best predicts unsuccessful tolvaptan therapy with a cut-off value of 46.5 $\mathrm{mEq} / \mathrm{g} \cdot \mathrm{Cr}$ (AUC 0.847, 95\% CI: 0.718-0.976, sensitivity 77\%, specificity 81\%, $P<0.01$ ). Double-positive results of $\mathrm{UNa} / \mathrm{UCr}<46.5 \mathrm{mEq} / \mathrm{g} \cdot \mathrm{Cr}$ and plasma BNP level $>778 \mathrm{pg} / \mathrm{mL}$ predicted unsuccessful tolvaptan therapy with high diagnostic accuracy (sensitivity $54 \%$, specificity $100 \%$, positive predictive value $100 \%$, negative predictive value $89 \%$, and accuracy 90\%). In summary, short-term tolvaptan therapy ameliorated HF symptoms and provided hemodynamic improvement in the majority of patients, and $\mathrm{UNa} / \mathrm{UCr}$ and $\mathrm{BNP}$ level strongly predicted the therapeutic outcomes. (Int Heart J 2016; 57: 211-219)
\end{abstract}

Key words: Vasopressin V2-receptor antagonist, Loop diuretics, Diuresis

$\mathrm{F}$ uid removal is an important component of heart failure (HF) treatment to reduce systemic and pulmonary congestion and relieve signs and symptoms of edema. Recent clinical and experimental studies have demonstrated that selective vasopressin V2 receptor antagonism by oral tolvaptan administration inhibits free water reabsorption in the kidney's collecting tubules, and therefore stabilizes hemodynamic state, ameliorates congestion and edema, and corrects hyponatremia. $^{1-10)}$ Successful oral tolvaptan therapy can support early ambulation, and can minimize the need for and duration of hospitalization.

An increment in urine volume has been recognized as the best indicator of clinical response to tolvaptan therapy in most studies $;^{3,6,11,12)}$ however, it has not been fully investigated whether increment of urine volume and resultant body weight loss achieved by V2 receptor antagonism always lead to symptom relief and sufficient hemodynamic improvement. ${ }^{13)}$ There- fore, we evaluated the effects of oral tolvaptan therapy on short-term clinical course by quantitatively assessing symptoms and hemodynamic states in patients with HF and volume overload. In addition, we identified the predictors of the therapeutic outcomes.

\section{MeTHODS}

Subjects: This clinical investigation was approved by the $\mathrm{Hu}-$ man Studies Subcommittee of Mie University Graduate School of Medicine (reference number: 2777). The study complies with the Declaration of Helsinki. All patients gave written informed consent. We retrospectively investigated 60 consecutive in-hospital patients with decompensated HF who received oral tolvaptan therapy for the treatment of volume overload between December 2010 and December 2014 in our cardiology

From the ${ }^{1}$ Department of Cardiology and Nephrology, Mie University Graduate School of Medicine, ${ }^{2}$ Clinical Research Support Center, Mie University Hospital, ${ }^{3}$ Department of Molecular and Laboratory Medicine, Mie University Graduate School of Medicine, Tsu, Japan.

Address for correspondence: Kaoru Dohi, MD, Department of Cardiology and Nephrology, Mie University Graduate School of Medicine, 2-174 Edobashi, Tsu 5148507, Japan. E-mail: dohik@ clin.medic.mie-u.ac.jp

Received for publication August 19, 2015. Revised and accepted October 20, 2015

Released in advance online on J-STAGE March 11, 2016.

All rights reserved by the International Heart Journal Association. 
ward. The HF symptoms of all patients worsened despite receiving HF treatment including oral diuretic therapy before hospital admission. Daily salt intake was limited to $6 \mathrm{~g} /$ day in all patients during hospitalization. Patients who required cardiac intensive care because of "non-ambulatory" New York Heart Association (NYHA) class IV HF including cardiogenic shock were not included.

Procedure: All patients underwent baseline blood and urine tests including neurohumoral assessment in the early morning before breakfast, chest X-rays, echocardiography, and hemodynamic measurements with right heart catheterization. Vital signs, 24-hour fluid intake, and urine volume were measured at baseline and every 24 hours thereafter. Body weight was measured after urination and before breakfast at baseline and every subsequent day during tolvaptan therapy. Estimated glomerular flow rate (eGFR) was calculated using an equation modified for the Japanese. ${ }^{14)}$ First-morning spot urine tests included the measurements of osmolality, sodium (UNa), and creatinine (UCr). Since we failed to measure urine urea nitrogen (UUN) in about half of the patients, the following formula was used to estimate UUN. ${ }^{15)}$

Urine osmolality $=1.07 \times\{2 \times[\mathrm{UNa}(\mathrm{mEq} / \mathrm{L})]+[\mathrm{UUN}$ $(\mathrm{mg} / \mathrm{dL})] / 2.8+[\mathrm{UCr}(\mathrm{mg} / \mathrm{dL})] \times 2 / 3\}+16$.

It was planned that all patients would undergo repeated blood and urine tests, chest X-rays, echocardiography, and right heart catheterization 7 days after the initiation of tolvaptan therapy. The HF symptom score was calculated as the summation of scores that were assigned to symptoms due to HF, such as 1) pitting edema in the lower extremities [1 point], 2) pulmonary congestion [1 point], 3) jugular venous distention [1 point], 4) dyspnea [1 point], and 5) degree of NYHA (1-4 points were assigned for each class, eg, 4 points were assigned for NYHA class IV) before and 7 days after tolvaptan therapy. ${ }^{3,16)}$

Neurohumoral assessment: Plasma B-type natriuretic peptide (BNP) and atrial natriuretic peptide (ANP) levels, plasma renin activity, plasma aldosterone concentration, and plasma arginine-vasopressin level were obtained after at least 15 minutes of rest in a sitting position, before drug ingestion, and before breakfast. Blood samples for the determination of plasma BNP and ANP levels were collected in a tube containing potassium ethylenediaminetetra-acetic acid, kept at room temperature, and analyzed within 4 hours of collection. Plasma renin activity, plasma aldosterone concentration, and plasma arginine-vasopressin level were measured using a radioimmunoassay at a commercial laboratory (SRL, Tokyo). ${ }^{5)}$

Echocardiographic assessment: Left ventricular end-diastolic dimension and end-systolic dimension were assessed from the parasternal long-axis view. Left ventricular ejection fraction was assessed using the biplane Simpson's rule.

Hemodynamic assessment: During right heart catheterization, mean pulmonary capillary wedge pressure (PCWP), mean right atrial pressure (RAP), systolic pulmonary artery pressure (PAP), mean PAP, diastolic PAP, and estimated cardiac output were recorded. . $^{\text {A }}$ Arm-cuff blood pressure measurements were performed simultaneously with right heart catheterization. The following formulas were used to calculate standard hemodynamic parameters derived from the above measurements.

Cardiac index $=$ cardiac output $/$ body surface area

Systemic vascular resistance index $=($ mean blood pressure - mean RAP) $\times 80 /$ cardiac index
Classification of successful and unsuccessful tolvaptan therapy: Patients were divided into two groups according to their clinical course during tolvaptan therapy. They were classified as being successfully treated (group 1) if they did not meet either of the following two criteria, and they were classified as being unsuccessfully treated (group 2) if they met at least one of them: 1) HF symptom score worsened or 2) the presence of a stationary HF symptom score $\geq 6$ points, and mean PCWP > $18 \mathrm{mmHg}$ and mean RAP $>10 \mathrm{mmHg}$, after tolvaptan therapy. Statistical analysis: All numerical data are expressed as the mean \pm standard deviation or median (interquartile range). Continuous data were compared by the unpaired $t$-test or nonparametric Mann-Whitney test depending on the outcome of tests for normality. Categorical data were assessed by the chisquare test. Two-way repeated measures analysis of variance was used to evaluate main (time; group) and interaction effects (time x group) for clinical, laboratory, and hemodynamic variables before and after tolvaptan therapy. Post hoc analysis was used for pre-post comparisons where either the main or the interaction effect was statistically significant. The area under the curve was calculated, and the optimal cut-off values of predictors of unsuccessful tolvaptan therapy were determined. Univariate and multivariate binary logistic analyses with a forward stepwise procedure $(P<0.10$ for entry) were applied to assess the relationships between predictors of unsuccessful tolvaptan therapy and clinical variables. $P$ values $<0.05$ were considered statistically significant. All statistical analyses were performed using SPSS 17.0 J (SPSS Japan Institute, Tokyo).

\section{RESUltS}

Clinical characteristics: Among all 60 patients, 18 (30\%) had NYHA class IV HF symptoms, but none of the 18 patients had orthopnea or severe hypoxemia with arterial oxygen saturation below $90 \%$ with oxygen supply via nasal cannula. The timing of tolvaptan administration was 3 (2-4) days after hospital admission. Among the 60 patients, 12 (20\%) received short-term or transient intravenous therapies including with loop diuretics (6 patients), carperitide (8 patients), and/or dobutamine (1 patient) infusion either alone or in combination for the initial treatment of HF upon hospital admission, but all were successfully discontinued before tolvaptan therapy. Fifty-nine patients received $7.5 \mathrm{mg} /$ day tolvaptan and only 1 patient received 15 $\mathrm{mg}$ /day tolvaptan. Among 18 patients with NYHA class IV HF symptoms, $61 \%$ were successfully treated with tolvaptan therapy. The majority of the 60 subjects $(78 \%)$ were successfully treated (group 1), but 13 patients (22\%) were unsuccessfully treated with tolvaptan therapy (group 2). Table I shows comparisons of the clinical characteristics between the two groups. The prevalence of patients with NYHA class IV, HF symptom score, and heart rate were higher in group 2 than in group 1. Blood pressure levels were similar in the two patient groups. Average dosages of furosemide-equivalent loop diuretics, enalapril-equivalent angiotensin converting enzyme inhibitors (ACEI)/angiotensin receptor blockers (ARB), and carvediol-equivalent beta-blockers were similar in the two patient groups. The time intervals from hospitalization to initiation of tolvaptan therapy were similar in the two groups. Table II shows comparisons of laboratory, echocardiographic, and hemodynamic parameters at baseline between the two groups. 
Table I. Baseline Clinical Characteristics

\begin{tabular}{|c|c|c|c|c|}
\hline & $\begin{array}{c}\text { All } \\
(n=60)\end{array}$ & $\begin{array}{l}\text { Group 1 } \\
(n=47)\end{array}$ & $\begin{array}{l}\text { Group 2 } \\
(n=13)\end{array}$ & $P$ \\
\hline \multicolumn{5}{|l|}{ Demographic parameters } \\
\hline Male, $\%$ & 77 & 77 & 77 & 0.980 \\
\hline Age, years & $70 \pm 11$ & $71 \pm 11$ & $66 \pm 11$ & 0.209 \\
\hline NYHA IV, $n(\%)$ & $18(30)$ & $11(23)$ & $7(54)$ & 0.036 \\
\hline HF symptom score & $6.5 \pm 1.3$ & $6.2 \pm 1.4$ & $7.3 \pm 0.8$ & 0.006 \\
\hline Body weight, $\mathrm{kg}$ & $63.1 \pm 14.1$ & $62.0 \pm 13.7$ & $67.2 \pm 14.8$ & 0.132 \\
\hline Systolic blood pressure, $\mathrm{mmHg}$ & $115 \pm 23$ & $117 \pm 24$ & $109 \pm 20$ & 0.346 \\
\hline Heart rate, bpm & $74 \pm 13$ & $71 \pm 12$ & $82 \pm 14$ & 0.009 \\
\hline Daily urine volume, $\mathrm{mL} /$ day & $1410 \pm 915$ & $1511 \pm 953$ & $1023 \pm 592$ & 0.049 \\
\hline Daily water intake, $\mathrm{mL} /$ day & $731 \pm 274$ & $749 \pm 292$ & $665 \pm 183$ & 0.341 \\
\hline Ischemic etiology, $\%$ & 37 & 31 & 38 & 0.621 \\
\hline Atrial fibrillation, \% & 52 & 53 & 46 & 0.656 \\
\hline \multicolumn{5}{|l|}{ Medication } \\
\hline ACEI/ARB, $\%$ & 88 & 91 & 77 & 0.151 \\
\hline Enalapril-equivalent doses, $\mathrm{mg}$ & $5(5-10)$ & $5(5-10)$ & $5(5-10)$ & 0.434 \\
\hline Beta-blockers, $\%$ & 58 & 57 & 62 & 0.793 \\
\hline Carvedilol-equivalent doses, mg & $2.5(0-5)$ & $2.5(0-5)$ & $2.5(0-5)$ & 0.895 \\
\hline Loop diuretics, \% & 97 & 96 & 100 & 0.453 \\
\hline Furosemide-equivalent doses, mg & $40(20-60)$ & $40(20-60)$ & $60(20-80)$ & 0.261 \\
\hline Thiazide diuretics, $\%$ & 10 & 11 & 8 & 0.756 \\
\hline Aldosterone antagonists, $\%$ & 58 & 60 & 54 & 0.713 \\
\hline Oral inotropic agents, $\%$ & 5 & 4 & 8 & 0.618 \\
\hline \multicolumn{5}{|l|}{ Tolvaptan therapy } \\
\hline Time from hospital admission, days & $3(2-4)$ & $3(2-4)$ & $3(2-4)$ & 0.860 \\
\hline Time from oral to RHC, days & $7(7-7)$ & $7(7-7)$ & $7(7-8)$ & 0.221 \\
\hline Duration, days & $10(7-19)$ & $8(7-15)$ & $17(8-45)$ & 0.010 \\
\hline \multicolumn{5}{|l|}{ Outcome } \\
\hline Hospitalization, days & $23(17-35)$ & $19(17-27)$ & $45(35-67)$ & $<0.001$ \\
\hline
\end{tabular}

NYHA indicates New York Heart Association; HF, heart failure; ACEI, angiotensin converting enzyme inhibitor; and ARB, angiotensin II receptor blocker.

Table II. Laboratory, Echocardiographic, and Hemodynamic Parameters

\begin{tabular}{|c|c|c|c|c|}
\hline & $\begin{array}{c}\text { All } \\
(n=60)\end{array}$ & $\begin{array}{l}\text { Group 1 } \\
(n=47)\end{array}$ & $\begin{array}{l}\text { Group 2 } \\
(n=13)\end{array}$ & $P$ \\
\hline \multicolumn{5}{|l|}{ Laboratory parameters } \\
\hline Blood urea nitrogen, $\mathrm{mg} / \mathrm{dL}$ & $29 \pm 15$ & $27 \pm 15$ & $35 \pm 14$ & 0.040 \\
\hline Serum creatinine, mg/dL & $1.37 \pm 0.68$ & $1.29 \pm 0.62$ & $1.64 \pm 0.80$ & 0.043 \\
\hline eGFR, $\mathrm{mL} /$ minute $/ 1.73 \mathrm{~m}^{2}$ & $46 \pm 19$ & $49 \pm 18$ & $38 \pm 16$ & 0.088 \\
\hline Serum sodium, mEq/L & $138 \pm 5$ & $138 \pm 4$ & $136 \pm 6$ & 0.318 \\
\hline Serum potassium, mEq/L & $4.1 \pm 0.6$ & $4.1 \pm 0.5$ & $4.0 \pm 0.8$ & 0.498 \\
\hline Serum osmolality, mOsm $/ \mathrm{kgH}_{2} \mathrm{O}$ & $289 \pm 11$ & $289 \pm 10$ & $289 \pm 12$ & 0.781 \\
\hline Plasma BNP, pg/mL & $629 \pm 508$ & $501 \pm 389$ & $1094 \pm 607$ & 0.002 \\
\hline Plasma ANP, pg/mL & $190 \pm 156$ & $169 \pm 152$ & $266 \pm 143$ & 0.025 \\
\hline Plasma arginine-vasopressin, $\mathrm{pg} / \mathrm{mL}$ & $7.0 \pm 7.0$ & $7.2 \pm 7.6$ & $6.6 \pm 4.2$ & 0.627 \\
\hline Plasma renin activity, $\mathrm{ng} / \mathrm{mL} / \mathrm{hour}$ & $5.5 \pm 6.5$ & $5.6 \pm 6.7$ & $5.4 \pm 5.7$ & 0.651 \\
\hline Plasma aldosterone, $\mathrm{pg} / \mathrm{mL}$ & $109 \pm 105$ & $105 \pm 98$ & $124 \pm 127$ & 0.848 \\
\hline Urine osmolality, $\mathrm{mOsm} / \mathrm{kgH}_{2} \mathrm{O}$ & $442 \pm 147$ & $436 \pm 151$ & $464 \pm 129$ & 0.499 \\
\hline $\mathrm{UNa} / \mathrm{UCr}, \mathrm{mEq} / \mathrm{g} \cdot \mathrm{Cr}$ & $104 \pm 79$ & $121 \pm 79$ & $44 \pm 40$ & $<0.001$ \\
\hline $\mathrm{UUN} / \mathrm{UCr}, \mathrm{mg} / \mathrm{g} \cdot \mathrm{Cr}$ & $6.6 \pm 2.5$ & $6.6 \pm 2.7$ & $6.5 \pm 1.7$ & 0.770 \\
\hline Fractional excretion of sodium, $\%$ & $1.1 \pm 1.5$ & $1.3 \pm 1.6$ & $0.6 \pm 0.5$ & 0.023 \\
\hline Fractional excretion of urine nitrogen, $\%$ & $33 \pm 16$ & $33 \pm 17$ & $30 \pm 8$ & 0.453 \\
\hline \multicolumn{5}{|l|}{ Echocardiographic parameters } \\
\hline Left ventricular diastolic diameter, $\mathrm{mm}$ & $60 \pm 13$ & $60 \pm 11$ & $62 \pm 17$ & 0.700 \\
\hline Ejection fraction, $\%$ & $40 \pm 19$ & $41 \pm 18$ & $36 \pm 21$ & 0.309 \\
\hline \multicolumn{5}{|l|}{ Hemodynamic parameters } \\
\hline Mean RAP, mmHg & $12 \pm 5$ & $11 \pm 5$ & $16 \pm 5$ & 0.006 \\
\hline Systolic PAP, mmHg & $50 \pm 15$ & $48 \pm 15$ & $54 \pm 13$ & 0.175 \\
\hline Mean PCWP, mmHg & $24 \pm 7$ & $23 \pm 8$ & $28 \pm 5$ & 0.008 \\
\hline Cardiac index, $\mathrm{L} /$ minute $/ \mathrm{m}^{2}$ & $2.4 \pm 0.7$ & $2.4 \pm 0.7$ & $2.2 \pm 0.7$ & 0.216 \\
\hline Systemic vascular resistance index, dynes $/ \mathrm{sec} / \mathrm{cm}^{-5} / \mathrm{m}^{2}$ & $2678 \pm 1040$ & $2654 \pm 962$ & $2779 \pm 1316$ & 0.726 \\
\hline
\end{tabular}

eGFR indicates estimated glomerular filtration rate; BNP, brain natriuretic peptide; ANP, atrial natriuretic peptide; UNa/UCr, urine sodium/creatinine ratio; UUN/UCr, urine urea nitrogen/creatinine ratio; RAP, right atrial pressure; PAP, pulmonary artery pressure; and PCWP, pulmonary capillary wedge pressure. 
Table III. Clinical Effects of Tolvaptan Therapy

\begin{tabular}{|c|c|c|c|c|c|}
\hline & $\begin{array}{l}\text { Group 1 } \\
(n=47)\end{array}$ & $\begin{array}{l}\text { Group 2 } \\
(n=13)\end{array}$ & $\begin{array}{l}\text { Group } \\
\text { effect } P\end{array}$ & $\begin{array}{l}\text { Time } \\
\text { effect } P\end{array}$ & $\begin{array}{c}\text { Interaction } \\
\text { effect } P\end{array}$ \\
\hline \multicolumn{6}{|l|}{ Daily urine volume, $\mathrm{mL} /$ day } \\
\hline Baseline (day 0) & $1511 \pm 957$ & $1023 \pm 592$ & 0.146 & $<0.001$ & 0.904 \\
\hline Post-treatment (day 1) & $2315 \pm 1523^{*}$ & $1786 \pm 1106^{*}$ & & & \\
\hline \multicolumn{6}{|l|}{ Daily water intake, $\mathrm{mL} /$ day } \\
\hline Baseline (day 0) & $749 \pm 292$ & $665 \pm 183$ & 0.332 & 0.002 & 0.799 \\
\hline Post-treatment (day 1) & $1114 \pm 726^{*}$ & $973 \pm 283$ & & & \\
\hline \multicolumn{6}{|l|}{ Body weight, $\mathrm{kg}$} \\
\hline Baseline (day 0) & $62.0 \pm 13.7$ & $67.2 \pm 14.8$ & 0.228 & $<0.001$ & 0.714 \\
\hline Post-treatment (day 7) & $59.6 \pm 13.5^{*}$ & $64.8 \pm 13.7^{*}$ & & & \\
\hline \multicolumn{6}{|l|}{ HF symptom score } \\
\hline Baseline (day 0) & $6.2 \pm 1.4$ & $7.3 \pm 0.8$ & $<0.001$ & $<0.001$ & $<0.001$ \\
\hline Post-treatment (day 7) & $2.8 \pm 1.0^{*}$ & $6.9 \pm 0.8$ & & & \\
\hline \multicolumn{6}{|l|}{ Blood urea nitrogen, $\mathrm{mg} / \mathrm{dL}$} \\
\hline Baseline (day 0) & $27 \pm 15$ & $35 \pm 14$ & 0.293 & 0.087 & 0.044 \\
\hline Post-treatment (day 7) & $32 \pm 16^{*}$ & $35 \pm 20$ & & & \\
\hline \multicolumn{6}{|l|}{ Serum creatinine, $\mathrm{mg} / \mathrm{dL}$} \\
\hline Baseline (day 0) & $1.29 \pm 0.62$ & $1.64 \pm 0.80$ & 0.201 & 0.121 & 0.127 \\
\hline Post-treatment (day 7) & $1.39 \pm 0.74$ & $1.64 \pm 0.92$ & & & \\
\hline \multicolumn{6}{|l|}{ Serum sodium, mEq/L } \\
\hline Baseline (day 0) & $138 \pm 4$ & $136 \pm 6$ & 0.214 & 0.137 & 0.889 \\
\hline Post-treatment (day 7) & $139 \pm 4$ & $137 \pm 6$ & & & \\
\hline \multicolumn{6}{|l|}{ Plasma BNP, pg/mL } \\
\hline Baseline (day 0) & $501 \pm 389$ & $1094 \pm 607$ & $<0.001$ & 0.182 & 0.016 \\
\hline Post-treatment (day 7) & $350 \pm 317^{*}$ & $1138 \pm 493$ & & & \\
\hline \multicolumn{6}{|l|}{ Plasma ANP, pg/mL } \\
\hline Baseline (day 0) & $169 \pm 152$ & $266 \pm 143$ & 0.004 & 0.642 & 0.002 \\
\hline Post-treatment (day 7) & $126 \pm 188^{*}$ & $329 \pm 179^{*}$ & & & \\
\hline
\end{tabular}

${ }^{*} P<0.05$ versus baseline. HF indicates heart failure; BNP, brain natriuretic peptide; and ANP, atrial natriuretic peptide.

Group 2 had higher blood urea nitrogen, serum creatinine, and plasma BNP and ANP levels than group 1, whereas sodium concentrations, plasma arginine-vasopressin levels, plasma renin activity, and plasma aldosterone concentration were similar in the two groups. Although the levels of urine osmolality were similar in the two groups, $\mathrm{UNa} / \mathrm{UCr}$ and fractional excretion of sodium were lower in group 2 than in group 1 . In contrast, UUN/UCr and fractional excretion of urine nitrogen were similar in the two groups. Group 2 had higher mean PCWP and mean RAP than group 1 at baseline, whereas cardiac index and systemic vascular resistance index were similar in the two groups.

Effects of tolvaptan therapy on clinical, laboratory, and hemodynamic parameters: Table III shows comparisons of the clinical effects of tolvaptan therapy between the two groups. Oral tolvaptan administration increased 24-hour urine volume at day 1 with no significant differences between the two groups. In addition, the averages of daily urine volume through days 1-7 were similar in the two groups (1987 \pm 890 and 1813 $\pm 978 \mathrm{~mL} /$ day, $P=\mathrm{NS})$. Body weight decreased in $94 \%$ of the patients in group 1 and in $92 \%$ of the patients in group 2 seven days after the initiation of tolvaptan therapy, with no significant differences between the two groups. However, the HF symptom score improved and plasma natriuretic peptide level decreased only in group 1 . The changes in serum creatinine levels (interaction effect $P=0.127$ ) were not significantly different between the two groups. Three patients in group 2 required additional interventions including intravenous infusion of inotropic agent and short-term ultrafiltration within 7 days after the initiation of tolvaptan therapy because of worsening of HF, and could not undergo follow-up hemodynamic measurement with right heart catheterization during tolvaptan therapy. In the other 57 patients, right heart catheterization was performed at a median of 7 (7-7) days after the initiation of tolvaptan therapy. Mean PCWP and mean RAP decreased only in group 1, with a significant interaction effect $(P<0.01$, each), whereas cardiac index and systemic vascular resistance index were unchanged after tolvaptan therapy in both groups (Figure 1). After the short-term tolvaptan therapy, only 1 of 47 patients in group 1 , but 12 out of 13 patients in group 2 required additional interventions including intravenous inotropic and/or carperitide therapy beyond simply maintaining the doses of oral diuretics during the hospital stay (Table IV). Hospital stay was significantly longer in group 2 than in group 1: 19 (17-27) versus 45 (35-67) days, $P<0.01$.

Predictors of successful tolvaptan therapy: The results of univariate and multivariate logistic regression analyses evaluating the clinical, laboratory, and hemodynamic risk factors for unsuccessful tolvaptan therapy are shown in Table V. Multivariate logistic regression analysis showed that plasma BNP level was positively and $\mathrm{UNa} / \mathrm{UCr}$ was negatively correlated to unsuccessful tolvaptan therapy. In contrast, serum sodium level, eGFR, urine osmolality, and all hemodynamic parameters were not independently correlated with the clinical outcomes of short-term tolvaptan therapy. Receiver operating characteristic curve analysis showed that $\mathrm{UNa} / \mathrm{UCr}$ best predicted unsuccessful tolvaptan therapy, with a cut-off value of $46.5 \mathrm{mEq} /$ g.Cr with an area under the curve of 0.847 and $95 \%$ confidence interval of $0.718-0.976$ (sensitivity $77 \%$, specificity $81 \%$, positive predictive value $53 \%$, negative predictive value $93 \%$, and 

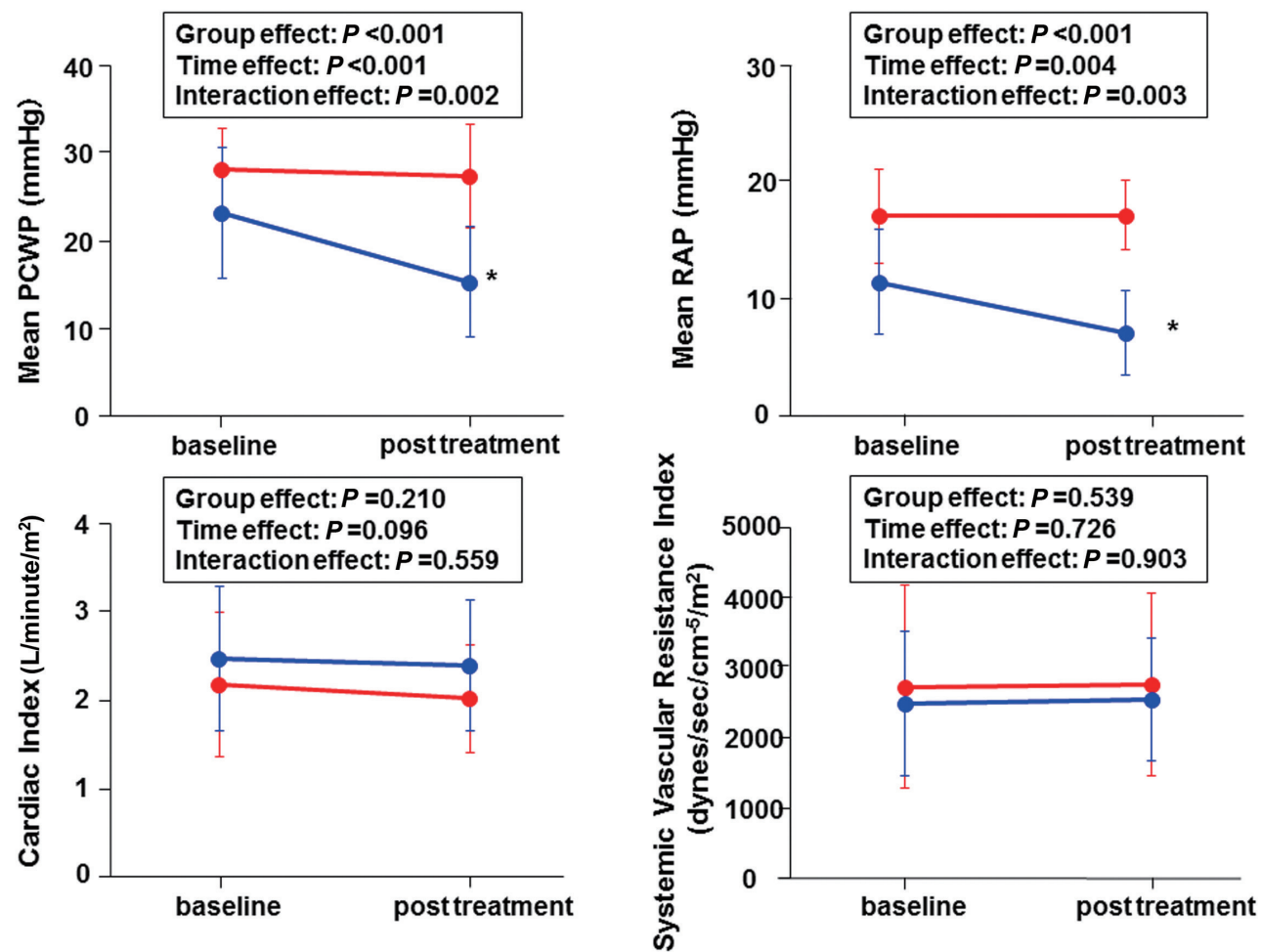

Figure 1. Comparisons of hemodynamic parameters at baseline and after tolvaptan treatment in group 1 (blue circle) and group 2 (red circles). PCWP indicates pulmonary capillary wedge pressure; RAP, right atrial pressure; SVRI, systemic vascular resistance index; and $\mathrm{CI}$, cardiac index. ${ }^{*} P<0.05$ versus baseline.

Table IV. Details of Additional Therapy for Patients in Group 2

\begin{tabular}{cccl}
\hline No & Age & $\begin{array}{c}\text { HF symptom score } \\
\text { at base line }\end{array}$ & Intervention \\
\hline 1 & 77 & 7 & Ultrafiltration \\
2 & 80 & 7 & Dobutamine + Carperitide \\
3 & 57 & 8 & Carperitide \\
4 & 47 & 8 & Milrinone + Furosemide \\
5 & 55 & 8 & Dobutamine + Carperitide + Furosemide \\
6 & 50 & 8 & Dobutamine + Milrinone + Carperitide \\
7 & 69 & 7 & Dobutamine + Carperitide + Catheter ablation for atrial flutter \\
8 & 56 & 5 & Dobutamine \\
9 & 81 & 7 & Dobutamine + Carperitide + Furosemide \\
10 & 75 & 7 & Dobutamine + Carperitide \\
11 & 70 & 7 & Dobutamine + Ultrafiltration \\
12 & 64 & 8 & Landiolol + Furosemide \\
13 & 78 & 7 & None \\
\hline
\end{tabular}

HF indicates heart failure.

accuracy $80 \%, P<0.01$, Figure 2 A), followed by plasma BNP level with a cut-off value of $778 \mathrm{pg} / \mathrm{mL}$ with an area under the curve of 0.786 and $95 \%$ confidence interval of $0.651-0.920$ (sensitivity $69 \%$, specificity $81 \%$, positive predictive value $50 \%$, negative predictive value $90 \%$, and accuracy $78 \%, P<$ $0.01)$. The combination of $\mathrm{UNa} / \mathrm{UCr}<46.5 \mathrm{mEq} / \mathrm{g} \cdot \mathrm{Cr}$ and plasma BNP level $>778 \mathrm{pg} / \mathrm{mL}$ predicted unsuccessful tolvaptan therapy with sensitivity of $54 \%$, specificity $100 \%$, positive predictive value $100 \%$, negative predictive value $89 \%$, and accuracy $90 \%$ (Figure 2B). The results of univariate and multivariate linear regression analyses of variables associated with $\mathrm{UNa} / \mathrm{UCr}$ are shown in Table VI. Plasma aldosterone concentration and dosage of oral furosemide were significantly and independently correlated with $\mathrm{UNa} / \mathrm{UCr}$.

\section{Discussion}

Tolvaptan therapy reduced body weight mainly via highvolume diuresis in almost all HF patients with volume overload in the present study. In addition, this therapy ameliorated HF symptoms and provided hemodynamic improvement in the majority of patients. However, about $20 \%$ of the patients were unsuccessfully treated, in whom 1) the HF symptom score 
Table V. Univariate and Multivariate Logistic Regression Analyses of the Factors for Unsuccessful Tolvaptan Therapy

\begin{tabular}{|c|c|c|c|c|}
\hline & \multicolumn{2}{|l|}{ Univariate } & \multicolumn{2}{|c|}{ Multivariate } \\
\hline & Odds ratio $(95 \% \mathrm{CI})$ & $P$ & Odds ratio $(95 \% \mathrm{CI})$ & $P$ \\
\hline NYHA, class & $3.784(1.230-11.645)$ & 0.003 & & \\
\hline HF symptom score & $2.447(1.195-5.012)$ & 0.014 & & \\
\hline Dosage of loop diuretic, mg & $1.016(0.996-1.036)$ & 0.113 & & \\
\hline Dosage of furosemide, $\mathrm{mg}$ & 1.019 (0.994-1.044) & 0.144 & & \\
\hline eGFR, $\mathrm{mL} /$ minute $/ 1.73 \mathrm{~m}^{2}$ & $0.967(0.931-1.005)$ & 0.086 & & \\
\hline Serum sodium, mEq/L & $0.931(0.825-1.050)$ & 0.245 & & \\
\hline Plasma BNP, pg/mL & $1.002(1.001-1.004)$ & 0.002 & $1.003(1.001-1.006)$ & 0.005 \\
\hline Plasma ANP, pg/mL & $1.004(1.000-1.008)$ & 0.072 & & \\
\hline Plasma arginine-vasopressin, $\mathrm{pg} / \mathrm{mL}$ & $0.986(0.895-1.087)$ & 0.781 & & \\
\hline Plasma renin activity, $\mathrm{ng} / \mathrm{mL} /$ hour & $0.995(0.901-1.099)$ & 0.916 & & \\
\hline Plasma aldosterone, pg/mL & $1.002(0.996-1.007)$ & 0.574 & & \\
\hline Urine osmolality, mOsm $/ \mathrm{kgH}_{2} \mathrm{O}$ & $1.001(0.997-1.006)$ & 0.547 & & \\
\hline $\mathrm{UNa} / \mathrm{UCr}, \mathrm{mEq} / \mathrm{g} \cdot \mathrm{Cr}$ & $0.971(0.950-0.992)$ & 0.007 & $0.959(0.929-0.990)$ & 0.009 \\
\hline Fractional excretion of sodium, $\%$ & $0.330(0.093-1.171)$ & 0.086 & & \\
\hline Ejection fraction, $\%$ & $0.986(0.952-1.020)$ & 0.408 & & \\
\hline Mean RAP, mmHg & $1.200(1.047-1.375)$ & 0.009 & & \\
\hline Mean PCWP, mmHg & $1.088(0.999-1.185)$ & 0.052 & & \\
\hline Cardiac index, $\mathrm{L} /$ minute $/ \mathrm{m}^{2}$ & $0.599(0.232-1.546)$ & 0.289 & & \\
\hline
\end{tabular}

NYHA indicates New York Heart Association; HF, heart failure; ACEI, angiotensin-converting enzyme inhibitor; ARB, angiotensin II receptor blocker; eGFR, estimated glomerular filtration rate; BNP, brain natriuretic peptide; ANP, atrial natriuretic peptide; $\mathrm{UNa} / \mathrm{UCr}$, urine sodium/creatinine ratio; RAP, right atrial pressure; and PCWP, pulmonary capillary wedge pressure.
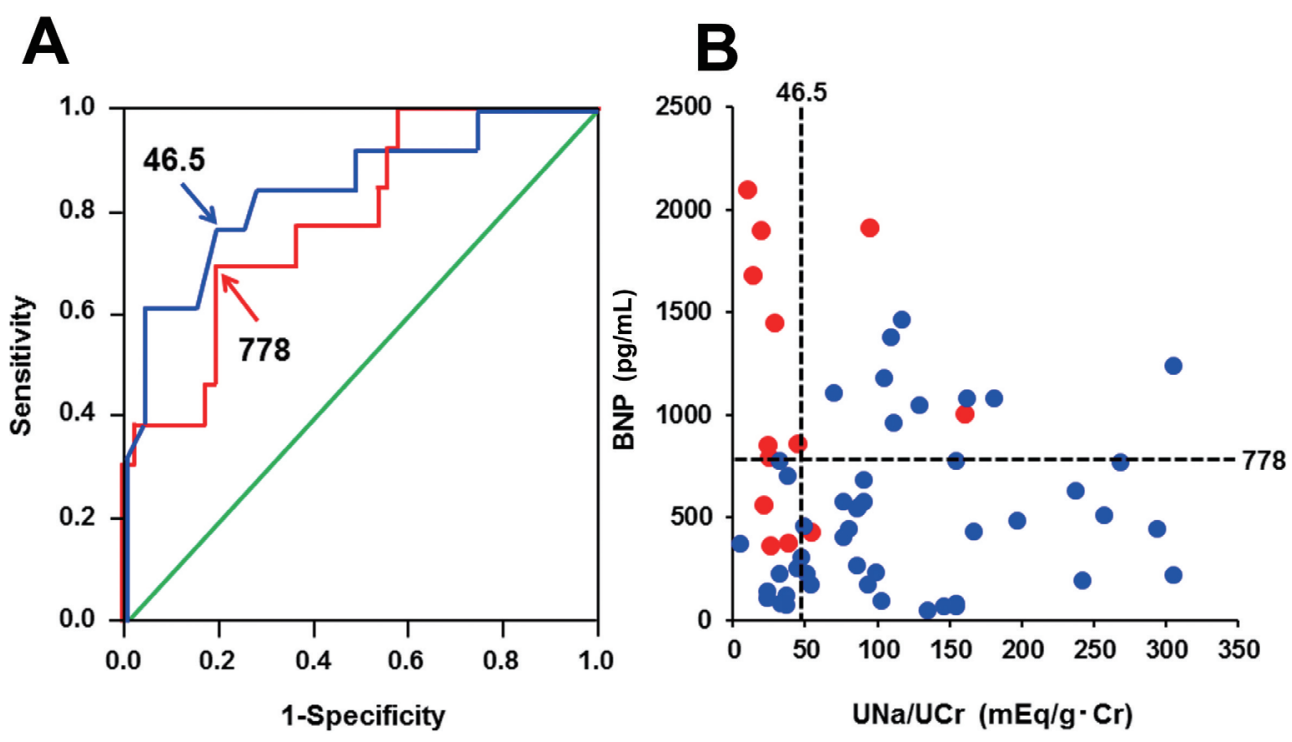

Figure 2. A: Receiver operating characteristic curve identifying cut-off values (arrows) of UNa/UCr (blue line) and plasma BNP level (red line) for the prediction of unsuccessful tolvaptan therapy. B: Scatter plots of UNa/UCr and plasma BNP levels in patients in group 1 (blue circle) and group 2 (red circles).

worsened or 2) there was the presence of a stationary HF symptom score $\geq 6$ points, and mean PCWP $>18 \mathrm{mmHg}$ and mean RAP > $10 \mathrm{mmHg}$, after tolvaptan therapy. Notably, the present study demonstrated that body weight reduction achieved by oral tolvaptan therapy itself was not always associated with symptom relief and sufficient hemodynamic improvement. We revealed that the combination of $\mathrm{UNa} / \mathrm{UCr}$ and plasma BNP level best predicted clinically unsuccessful tolvaptan therapy.

Recent clinical reports have demonstrated that the early use of oral tolvaptan in patients with HF and overt volume overload can effectively reduce water retention via high-volume diuresis, which can minimize the need for continuous intravenous HF therapy and support early ambulation. Indeed, Suzuki, et al recently demonstrated that tolvaptan was as effective as and safer than intravenous carperitide, a natriuretic peptide, by head-to-head comparison in a Japanese population. ${ }^{6}$ However, it is not fully understood whether successful volume depletion with tolvaptan therapy always leads to the amelioration of patient symptoms and provides hemodynamic improve- 
Table VI. Univariate and Multivariate Linear Regression Analyses of Variables Associated with UNa/UCr

\begin{tabular}{|c|c|c|c|c|}
\hline & \multicolumn{2}{|c|}{ Univariate } & \multicolumn{2}{|c|}{ Multivariate } \\
\hline & Coefficient & $P$ & $\beta$-Coefficient & $P$ \\
\hline NYHA, class & -0.039 & 0.768 & & \\
\hline HF symptom score & -0.095 & 0.469 & & \\
\hline Dosage of loop diuretic, mg & 0.032 & 0.810 & & \\
\hline Dosage of furosemide, mg & -0.345 & 0.007 & -0.322 & 0.009 \\
\hline $\mathrm{eGFR}, \mathrm{mL} / \mathrm{minute} / 1.73 \mathrm{~m}^{2}$ & -0.238 & 0.066 & & \\
\hline Serum sodium, mEq/L & 0.407 & 0.001 & & \\
\hline Plasma BNP, pg/mL & -0.024 & 0.854 & & \\
\hline Plasma ANP, pg/mL & 0.016 & 0.905 & & \\
\hline Plasma arginine-vasopressin, $\mathrm{pg} / \mathrm{mL}$ & 0.035 & 0.798 & & \\
\hline Plasma renin activity, ng/mL/hour & -0.216 & 0.104 & & \\
\hline Plasma aldosterone, $\mathrm{pg} / \mathrm{mL}$ & -0.388 & 0.003 & -0.390 & 0.002 \\
\hline Ejection fraction, $\%$ & 0.388 & 0.003 & & \\
\hline Mean RAP, mmHg & 0.011 & 0.935 & & \\
\hline Mean PCWP, mmHg & -0.209 & 0.109 & & \\
\hline Cardiac index, $\mathrm{L} /$ minute $/ \mathrm{m}^{2}$ & 0.159 & 0.226 & & \\
\hline
\end{tabular}

$\mathrm{UNa} / \mathrm{UCr}$ indicates urine sodium/creatinine ratio; NYHA, New York Heart Association; HF, heart failure; ACEI, angiotensin-converting enzyme inhibitor; ARB, angiotensin II receptor blocker; eGFR, estimated glomerular filtration rate; BNP, brain natriuretic peptide; ANP, atrial natriuretic peptide; RAP, right atrial pressure; and PCWP, pulmonary capillary wedge pressure.

ment, ${ }^{13)}$ especially in patients who are not concurrently treated with intravenous inotropic agents or intravenous diuretic/vasodilation therapy. In addition, it has not been elucidated how we can predict patients who will or will not be successfully treated with oral tolvaptan administration alone. Almost all patients achieved body weight reduction mainly via high-volume diuresis, and about $80 \%$ of all patients were successfully treated with oral tolvaptan therapy. Notably, about $60 \%$ of patients with ambulatory NYHA class IV were successfully treated with oral tolvaptan administration alone. However, about $20 \%$ of all patients had an unsuccessful clinical course during oral tolvaptan therapy despite achieving similar urine volume increment and body weight reduction to those with successful clinical outcomes. On the basis of these observations, tolvaptan can induce forced diuresis in the majority of HF patients unless they present with non-ambulatory NYHA class IV or cardiogenic shock; however, forced diuresis itself may not always result in sufficient cardiorenal hemodynamic improvement or symptom relief. Indeed, patients who were unsuccessfully treated with tolvaptan therapy did not show any improvements in hemodynamic parameters and HF symptom scores or plasma natriuretic peptide levels. Therefore, it is very important to identify the risks for unsuccessful oral tolvaptan therapy in order to develop personalized treatment plans. Patients who were unsuccessfully treated with tolvaptan therapy had clinical conditions with severely decompensated HF and might have required prompt and specific cardiorenal support mainly with intravenous inotropic and/or carperitide therapy concomitantly with aquaretic therapy upon hospital admission.

We revealed that first-morning spot $\mathrm{UNa} / \mathrm{UCr}$ best predicts successful tolvaptan therapy in patients with HF and clinical evidence of volume overload. Since $\mathrm{UCr}$ is inversely proportional to urine flow rate, $\mathrm{UNa} / \mathrm{UCr}$ accounts for variations in urine flow rate, ${ }^{17)}$ assuming no effect of urine flow rate on urine sodium or creatinine excretion rate. HF-related relative arterial underfilling is an important signal that triggers sodium and water retention. ${ }^{18-20)}$ It stimulates the renin-angiotensin-aldosterone system (RAAS) and sympathetic nervous system
(SNS), which largely contribute to renal tubular sodium reabsorption to maintain an effective arterial blood volume. In a clinical setting, loop diuretic-induced sodium loss in the daytime without sufficient RAAS blockade may further augment renal tubular sodium reabsorption at night. Indeed, plasma aldosterone concentration and the dosage of furosemide were independently associated with first-morning spot UNa/UCr. Interestingly, fractional excretion of urine nitrogen, a marker of prerenal azotemia, was similar in the two groups. Therefore, we speculate that low first-morning spot $\mathrm{UNa} / \mathrm{UCr}$ is a clinical indicator of intrinsic RAAS/SNS activation secondary to arterial underfilling rather than true hypovolemia. The combination of BNP, which reflects the severity of ventricular overload, and $\mathrm{UNa} / \mathrm{UCr}$ provided further improvement of accuracy in the prediction of clinically unsuccessful tolvaptan therapy. Low UNa/UCr and high plasma BNP levels may reflect HFrelated arterial underfilling and compensatory processes of maximal renal tubular sodium reabsorption despite excessive cardiac overload, indicating severe decompensated HF. In such clinical conditions, forced diuresis by tolvaptan may augment renal underfilling and may result in insufficient cardiorenal hemodynamic improvement.

Right heart catheterization-derived intra-cardiac pressure did not decrease only in the patient group that was unsuccessfully treated with tolvaptan therapy, even though there was a significant reduction in body weight. Therefore, we speculate that the central fluid shift from the venous capacitance vessels of the splanchnic circulation may play a role in persistent excessive cardiac preload in such patients via the activation of RAAS and SNS. ${ }^{21)}$ Large numbers of $\alpha 1$ and $\alpha 2$ adrenergic receptors are present in the splanchnic veins, making them highly sensitive to stimulation by the SNS. ${ }^{21)}$ Although the dosages of ACEI/ARB and beta blockers were similar in the two patient groups, sufficient dosages of these medications for effectively regulating RAAS and SNS can vary individually depending on their clinical conditions including HF severity and the presence of coexisting disorders. Intrinsic RAAS and SNS might be much more activated and/or insufficiently inhibited at 
baseline in patients who were not successfully treated with tolvaptan while plasma levels of renin activity and aldosterone were not statistically different between the two groups. The mechanisms underlying the discrepancy between the changes in body fluid and hemodynamic status warrant further investigation.

Potential limitations of the present study include the small sample population and the heterogeneous etiologies of HF with retrospective evaluation. Further studies with larger populations are needed to specify the etiological differences that might contribute to the effectiveness of tolvaptan therapy. Patients who required cardiac intensive care because of "non-ambulatory" NYHA class IV HF including cardiogenic shock were not included in the present study. In addition, as a singlecenter study with no control group for comparison, the generalizability of the present results is limited. Recent clinical observations including a post-marketing survey demonstrated that about $20 \%$ of patients received intravenous carperitide infusion concomitantly with oral tolvaptan therapy. ${ }^{4)}$ However, carperitide was not concomitantly administered with tolvaptan therapy in all patients in the present study. Three out of 13 patients in group 2 required additional interventions including intravenous infusion of inotropic agent and short-term ultrafiltration within 7 days after the initiation of tolvaptan therapy because of worsening of HF; therefore, the post-treatment data including the HF score, urine volume, body weight, renal function, and neurohumoral parameters in group 2 can be influenced by these therapies. Although almost all patients received $7.5 \mathrm{mg}$ of tolvaptan daily, the dose effects of this compound should be investigated further. Plasma levels of angiotensin II and catecholamines, which can mainly and directly contribute to renal tubular sodium reabsorption and the tones of venous capacitance vessels of the splanchnic circulation to maintain an effective arterial blood volume, were not measured in the present study. Also, the long-term effects of tolvaptan therapy in clinical responders in this population were not tested. Finally, the effects of tolvaptan treatment in patients with moderate to severe renal dysfunction warrant further evaluation. ${ }^{22)}$

Conclusions: Tolvaptan therapy provided body weight reduction mainly via high-volume diuresis in most patients, and about $80 \%$ of the patients were identified as clinical responders. However, this therapy was insufficient for symptom relief and hemodynamic improvement in a minority of the patients. The combination of low $\mathrm{UNa} / \mathrm{UCr}<46.5 \mathrm{mEq} / \mathrm{g} \cdot \mathrm{Cr}$ and high plasma BNP > $778 \mathrm{pg} / \mathrm{mL}$ can effectively predict successful tolvaptan therapy, and the measurements of these parameters that are easily performed by routine tests can help clinicians tailor HF treatment.

\section{ACKNowledgments}

We sincerely thank all of the patients, collaborating physicians, and other medical staff for their important contributions to this study.

\section{Disclosures}

Competing interests: K.D. received lecture fees of equal to or more than 500,000 yen from Otsuka Pharma Inc. in 2014. N.Y. received lecture fees of more than 500,000 yen from Daiichi Sankyo Co. Ltd. and Astellas Pharma Inc. in 2014. M.I. received lecture fees of more than 500,000 yen from Daiichi Sankyo Co. Ltd. in 2014. M.I. received a single-year unrestricted research grant for the Department of Cardiology and Nephrology, Mie University Graduate School of Medicine, of equal to or more than 2 million yen, from Astellas Pharma Inc., Pfizer Inc., Takeda Pharmaceutical Co. Ltd., Daiichi Sankyo Co. Ltd., Sumitomo Dainippon Pharma Co. Ltd. and Public Health Research Foundation in 2014.

The authors state there are no relevant conflicts of interest that should be reported.

\section{REFERENCES}

1. Onogawa T, Sakamoto Y, Nakamura S, Nakayama S, Fujiki H, Yamamura Y. Effects of tolvaptan on systemic and renal hemodynamic function in dogs with congestive heart failure. Cardiovasc Drugs Ther 2011; 25: S67-76.

2. Matsue Y, Suzuki M, Seya M, et al. Tolvaptan reduces the risk of worsening renal function in patients with acute decompensated heart failure in high-risk population. J Cardiol 2013; 61: 169-74.

3. Imamura T, Kinugawa K, Shiga T, et al. Novel criteria of urine osmolality effectively predict response to tolvaptan in decompensated heart failure patients--association between non-responders and chronic kidney disease. Circ J 2013; 77: 397-404.

4. Kinugawa K, Sato N, Inomata T, Shimakawa T, Iwatake N, Mizuguchi K. Efficacy and safety of tolvaptan in heart failure patients with volume overload. Circ J 2014; 78: 844-52.

5. Watanabe K, Dohi K, Sugimoto T, et al. Short-term effects of lowdose tolvaptan on hemodynamic parameters in patients with chronic heart failure. J Cardiol 2012; 60: 462-9.

6. Suzuki S, Yoshihisa A, Yamaki T, et al. Acute heart failure volume control multicenter randomized (AVCMA) trial: comparison of tolvaptan and carperitide. J Clin Pharmacol 2013; 53: 1277-85.

7. Perucca J, Bichet DG, Bardoux P, Bouby N, Bankir L. Sodium excretion in response to vasopressin and selective vasopressin receptor antagonists. J Am Soc Nephrol 2008; 19: 1721-31.

8. Shirakabe A, Hata N, Yamamoto M, et al. Immediate administration of tolvaptan prevents the exacerbation of acute kidney injury and improves the mid-term prognosis of patients with severely decompensated acute heart failure. Circ J 2014; 78: 911-21.

9. Ishikawa M, Kobayashi N, Sugiyama F, Onoda S, Ishimitsu T. Renoprotective effect of vasopressin v2 receptor antagonist tolvaptan in Dahl rats with end-stage heart failure. Int Heart J 2013; 54: 98-106.

10. Imamura T, Kinugawa $\mathrm{K}$, Minatsuki S, et al. Urine sodium excretion after tolvaptan administration is dependent upon baseline serum sodium levels: a possible explanation for the improvement of hyponatremia with scarce chance of hypernatremia by a vasopressin receptor antagonist. Int Heart J 2014; 55: 131-7.

11. Inomata T, Izumi T, Matsuzaki M, Hori M, Hirayama A. Phase III clinical pharmacology study of tolvaptan. Cardiovasc Drugs Ther 2011; 25: S57-65.

12. Matsuzaki M, Hori M, Izumi T, Asanoi H, Tsutamoto T. Effects of tolvaptan on volume overload in japanese patients with heart failure: results of a phase II, multicenter, randomized, double-blind, placebo-controlled, parallel-group study. Cardiovasc Drugs Ther 2011; 25: S19-31.

13. Toda H, Nakamura K, Nakahama M, et al; Tolvaptan Registry Investigators. Clinical characteristics of responders to treatment with tolvaptan in patients with acute decompensated heart failure: Importance of preserved kidney size. J Cardiol 2016; 67: 177-83.

14. Imai E, Horio M, Nitta K, et al. Modification of the modification of diet in renal disease (MDRD) study equation for japan. Am J Kidney Dis 2007; 50: 927-37. 
15. Imamura T, Kinugawa K, Minatsuki S, et al. Urine osmolality estimated using urine urea nitrogen, sodium and creatinine can effectively predict response to tolvaptan in decompensated heart failure patients. Circ J 2013; 77: 1208-13.

16. Imamura T, Kinugawa K, Minatsuki S, et al. Tolvaptan can improve clinical course in responders. Int Heart J 2013; 54: 377-81.

17. Waikar SS, Sabbisetti VS, Bonventre JV. Normalization of urinary biomarkers to creatinine during changes in glomerular filtration rate. Kidney Int 2010; 78: 486-94.

18. Sica DA. Sodium and water retention in heart failure and diuretic therapy: basic mechanisms. Cleve Clin J Med 2006; 73: S2-7.
(Review)

19. Schrier RW. Diagnostic value of urinary sodium, chloride, urea, and flow. J Am Soc Nephrol 2011; 22: 1610-3. (Review)

20. Schrier RW, Abraham WT. Hormones and hemodynamics in heart failure. N Engl J Med 1999; 341: 577-85. (Review)

21. Fallick C, Sobotka PA, Dunlap ME. Sympathetically mediated changes in capacitance: Redistribution of the venous reservoir as a cause of decompensation. Circ Heart Fail 2011; 4: 669-75.

22. Sato E, Nakamura T, Amaha M, et al. Effect of tolvaptan in patients with chronic kidney disease due to diabetic nephropathy with heart failure. Int Heart J 2014; 55: 533-8. 